\title{
ON THE ZEROS OF RATIONAL FUNCTIONS HAVING PRESCRIBED POLES, WITH APPLICATIONS TO THE DERIVATIVE OF AN ENTIRE FUNCTION OF FINITE GENRE
}

\author{
BY

\section{MORRIS MARDEN}

1. Introduction. Biernacki( ${ }^{(1)}$ and Dieudonné( $\left.{ }^{2}\right)$ have proved that, if $A$ is an arbitrary complex number, if $m_{1}, m_{2}, \cdots, m_{n}$ are positive integers, and if all the points $z_{1}, z_{2}, \cdots, z_{n}$ lie in a circle $C$ of radius $r$, then at least $n-1$ zeros of the function $F(z)=A+\sum_{j=1}^{n} m_{j} /\left(z-z_{j}\right)$ lie in the concentric circle of radius $2^{1 / 2} r$. This theorem was recently shown by $\mathrm{Nagy}\left({ }^{3}\right)$ to be valid also when the $m_{j}$ are arbitrary positive numbers.

In the present paper the theorem will be given a three-way generalization. The circle $C$ will be replaced by an arbitrary convex region, the constant $A$ by an arbitrary polynomial and the positive real $m_{j}$ by certain complex $m_{j}$. That is, the theorem will be generalized to functions of the form

$$
F(z)=\sum_{j=0}^{p-1} A_{j} z^{j}+\sum_{j=1}^{n} m_{j} /\left(z-z_{j}\right),
$$

where the $A_{j}$ are arbitrary complex numbers, where the $m_{j}$ are also complex numbers, and where the $z_{j}$ are points in a convex region $K$. The principal result on rational functions may be stated as follows.

THEOREM (1.1). Let $F(z)$ be a rational function which has at infinity a pole of order $p-1$ and has at the $n$ finite points $z_{j}(j=1,2, \cdots, n)$ simple poles with residues $m_{j}$ such that

$$
a \leqq \arg m_{j} \leqq a+\mu<a+\pi, \quad j=1,2, \cdots, n .
$$

Let $K$ be the smallest closed convex region conlaining all the $z_{j}$. Then $F(z)$ has at most $p$ zeros (counted with their multiplicities) exterior to the closed region $S(K, \psi)$ comprised of all points from which $K$ subtends an angle of at least $\psi=(\pi-\mu) /(p+1)$.

Presented to the Society, April 17, 1948, under the title On the zeros of rational functions with prescribed poles; received by the editors May 7, 1948.

(1) M. Biernacki, Sur les équations algébriques contenant des paramètres arbitraires, Akademija Umiejętnosii, Krak6w, Bulletin International ser. A (1927) pp. 541-685.

(2) J. Dieudonné, Sur quelques points de la théorie des zéros des polynômes, Bull. Sci. Math. (2) vol. 58 (1934) pp. 273-296.

(3) G. v. S. Nagy, Ein elementargeometrischer Satz und seine Anwendungen in der Geometrie der Polynome, Mathematischer und Naturwissenschaf tlicher Anzerger der Ungarischen Akademie der Wissenschaften vol. 61 (1942) pp. 776-785. 
Thus, if $K$ is a circle of radius $r, S(K, \psi)$ is the concentric circle of radius $r \csc (\psi / 2)$. If $K$ is a line-segment $A B$ of length $2 \mathrm{a}, S(K, \psi)$ is bounded by the two arcs of circles which pass through $A$ and $B$ and the points on the perpendicular bisector of $A B$ at a distance $[a \cot (\psi / 2)]$ from $A B$. In general, the region $S(K, \psi)$ is not convex, but it always is star-shaped with respect to $K\left({ }^{4}\right)$.

By the methods to be used in proving Theorem (1.1), a similar theorem will also be established for the zeros of meromorphic functions of the form

$$
\Phi(z)=P(z)+\left[m_{0} /\left(z-z_{0}\right)\right]+\sum_{j=1}^{\infty} m_{j} \phi\left(z, z_{j}\right),
$$

where $P(z)$ is an arbitrary polynomial of degree $p$ and $\phi(z, c)=(z-c)^{-1}$ $+\sum_{\substack{q=1 \\ k=0}} z^{k} / c^{k+1}, q \leqq p$. The latter theorem will be applied to the derivative of entire functions of genre $p$

$$
E(z)=e^{P(z)} z^{r} \prod_{j=1}^{\infty}\left\{\left[1-\left(z / z_{j}\right)\right] \exp \sum_{k=1}^{q}(1 / k)\left(z / z_{j}\right)^{k}\right\} .
$$

The location of the zeros of $E^{\prime}(z)$ relative to the zeros of $E(z)$ will be determined independently of $P(z)$. Thus, for example, the Lucas Theorem on the zeros of the derivative of a polynomial will be generalized to entire functions, without the restrictions which some previous investigators had found necessary to impose upon $p$ and $P(z)(5)$.

Incidentally, a new representation will be given for $E^{\prime}(z)$ in terms of any $p$ of its zeros and of all the zeros of $E(z)$, and will be used as the basis of an elementary proof of a classical theorem of Laguerre and Borel on the zeros of $E^{\prime}(z)$.

2. Some preliminary theorems. For the purpose of establishing Theorem (1.1), it will be convenient to define the two sets of symmetric functions of the variables $Z_{1}, Z_{2}, \cdots, Z_{p+1}$. Let $k \leqq q \leqq p+1$ and

$$
\begin{aligned}
f(k, q) & =(-1)^{k-1} \sum_{j=1}^{n}\left[m_{j} /\left(Z_{1}-z_{j}\right) \cdots\left(Z_{k-1}-z_{j}\right)\left(Z_{q}-z_{j}\right)\right] \\
S_{r}(k, q) & =\sum Z_{1}^{j_{1}} Z_{2}^{j_{2}} \cdots Z_{k-1}^{j_{k-1}} Z_{q}^{j_{k}},
\end{aligned}
$$

the latter sum consisting of all possible products with each $j=0,1, \cdots, r$ and $j_{1}+j_{2}+\cdots+j_{k}=r$. As one may easily verify, these functions have the properties

$$
f(k, q)-f(k, k)=\left(Z_{q}-Z_{k}\right) f(k+1, q),
$$

${ }^{4}$ ) Cf. discussion of regions $S(K, \psi)$ on pp. 20-23 of M. Marden, The geometry of the zeros of a polynomial in a complex variable, Mathematical Surveys, no. 3, New York, American Mathematical Society, 1949.

(5) Cf. the report on the zeros of entire functions in E. B. Van Vleck, On the location of roots of polynomials and entire functions, Bull. Amer. Math. Soc. vol. 35 (1929) pp. 643-683. 


$$
S_{r}(k, q)-S_{r}(k, k)=\left(Z_{q}-Z_{k}\right) S_{r-1}(k+1, q) .
$$

When the $Z_{j}$ are chosen as zeros of the function $F(z)$, the following result holds $\left({ }^{6}\right)$.

THEOREM (2.1) If $Z_{1}, Z_{2}, \cdots, Z_{p+1}$ are any $p+1$ distinct zeros of the function $F(z)$ defined by equation (1.1), then $f(p+1, p+1)=0$. That is,

$$
\sum_{j=1}^{n}\left[m_{j} /\left(Z_{1}-z_{j}\right)\left(Z_{2}-z_{j}\right) \cdots\left(Z_{p+1}-z_{j}\right)\right]=0 .
$$

For, by hypothesis, the $p$ coefficients $A_{j}$ in equation (1.1) satisfy the $p+1$ equations

$$
F\left(Z_{j}\right)=0, \quad j=1,2, \cdots, p+1 .
$$

It follows that the determinant

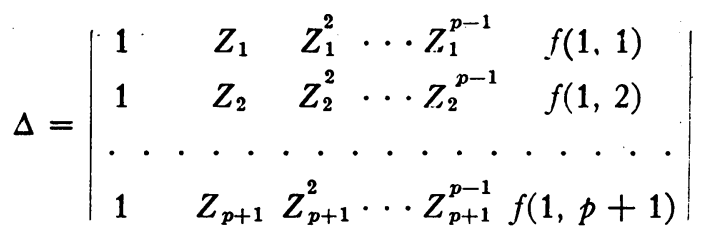

must vanish. As a first step in the evaluation of $\Delta$, its first row may be subtracted from the remaining rows and the common factor $\left(Z_{2}-Z_{1}\right)\left(Z_{3}-Z_{1}\right) \ldots$ $\left(Z_{p+1}-Z_{1}\right)$ removed. The result thereby obtained is the special case $r=1$ of the formula

$$
\Delta=\Delta_{r+1} \prod_{i=1}^{r} \prod_{j=i+1}^{p+1}\left(Z_{j}-Z_{i}\right)
$$

where $\Delta_{r}$ is the determinant

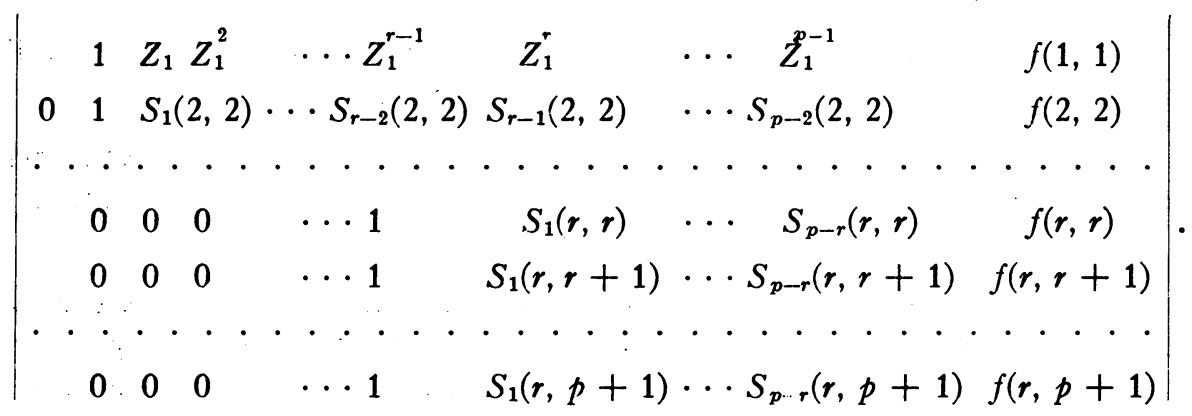

If in the determinant $\Delta_{r}$ the $r$ th row is subtracted from each row below it,

(6) Cf. the method in M. Marden, Kakeya's problem on the zeros of the derivative of a polynomial, Trans. Amer. Math. Soc. vol. 45 (1939) pp. 355-368. Also cf. J. C. Montgomery, The roots of a polynomial and its derivative, Bull. Amer. Math. Soc. vol. 47 (1941) pp. 621-624. See also pp. 89-90 of the book referred to in footnote 4 . 
the relation

$$
\Delta_{r}=\Delta_{r+1} \prod_{j=r+1}^{p+1}\left(Z_{j}-Z_{r}\right)
$$

is obtained, thus completing the proof of equation (2.6) by mathematical induction. The fact that $\Delta_{p+1}=f(p+1, p+1)$ leads now to the result

$$
\Delta=f(p+1, p+1) V(p+1)
$$

where $V(p+1)=\prod_{i=1}^{p} \prod_{j=i+1}^{p+1}\left(Z_{j}-Z_{i}\right)$, the latter being the Vandermonde determinant or discriminant of the $Z_{j}$.

If now the assumption that the $Z_{j}$ are distinct zeros of $F(z)$ is used, the corresponding $f(p+1, p+1)=0$ as required in Theorem (2.1).

Incidentally, from the above reasoning, the following result may be deduced.

TheOREM (2.2). If $Z_{1}, Z_{2}, \cdots, Z_{p}$ are any $p$ distinct zeros of the function $F(z)$ defined by equation (1.1), then

$$
F(z)=\prod_{j=1}^{p}\left(Z_{j}-z\right) \sum_{j=1}^{n}\left[m_{j} /\left(z-z_{j}\right)\left(Z_{1}-z_{j}\right) \cdots\left(Z_{p}-z_{j}\right)\right] .
$$

This result, somewhat more general than Theorem (2.1), may be derived by replacing the $(p+1)$ th equation in the system $(2.5)$ by the equation obtained by transposing $F(z)$ to the right side of equation (1.1). The only change required in the preceding formulas is therefore that of setting $Z_{p+1}=z$ and replacing the last element in the last row of $\Delta$ by $[f(1, p+1)-F(z)]$. The new

$$
\Delta=\left\{f(p+1, p+1)-\left[F(z) /\left(z-Z_{1}\right) \cdots\left(z-Z_{p}\right)\right]\right\} V(p+1)=0
$$

as required in Theorem (2.2).

Finally, the restriction in Theorem (2.1) that the zeros $Z_{j}$ be distinct will be removed by establishment of the following theorem.

TheOREM (2.3). Theorems (2.1) and (2.2) are valid even if not all the $Z_{j}$ are distinct provided no $Z_{j}$ occurs more oflen in (2.4) and (2.8) than its multiplicity as a sero of $F(\approx)$.

For example, let $Z_{1}=Z_{2}=\cdots=Z_{k}$ where $k$ is an integer not exceeding the multiplicity of $Z_{1}$ as a zero of $F(z)$, but assume that the remaining $Z_{j}$ are distinct from one another and from $Z_{1}$. In this case the first $k$ equations of the system (2.5) must be replaced by the equations

$$
F^{(i)}\left(Z_{1}\right)=0, \quad i=0,1, \cdots, k-1 .
$$

The corresponding determinant $\Delta^{\prime}$ may, however, be obtained from $\Delta$ by a limit process. Namely, 


$$
\Delta^{\prime}=\lim _{Z_{k \rightarrow Z_{1}}} \lim _{Z_{k \rightarrow 1^{-} \rightarrow Z_{1}}} \cdots \lim _{Z_{2} \rightarrow Z_{1}}[\Delta / V(k)] .
$$

From equation (2.6) it follows that $\Delta^{\prime}=f(p+1, p+1) V_{1}(p+1)$ where $V_{1}(p+1)$ is the Vandermonde determinant formed from only the distinct $Z_{j}$ in the sequence $Z_{1}, Z_{2}, \cdots, Z_{p+1}$.

3. Proof of Theorem (1.1). Theorems (2.1) and (2.3) will now be applied to the proof of Theorem (1.1). If $F(z)$ has at least $p+1$ zeros outside of $S(K, \psi)$, these zeros may be chosen as the $Z_{1}, Z_{2}, \cdots, Z_{p+1}$ occurring in equation (2.4). By the definition of $S(K, \psi), K$ subtends at each $Z_{j}$ an angle less than $\psi$. That is, corresponding to each $Z_{k}$, a point $t_{k} \neq Z_{k}$ may be found in $K$ such that

$$
0 \leqq \arg \left[\left(Z_{k}-t_{k}\right) /\left(Z_{k}-z\right)\right]<\psi
$$

for all points $z$ in $K$. In particular, since by hypothesis the points $z_{j}$ all lie in $K$, the vector $w_{j}=\prod_{k=1}^{p+1}\left[\left(Z_{k}-t_{k}\right) /\left(Z_{k}-z_{j}\right)\right]$ has the properties that $w_{j} \neq 0$ and

$$
0 \leqq \arg w_{j}<(p+1) \psi, \quad j=1,2, \cdots, n .
$$

Taken together with inequality (1.2), this leads to the conclusion

$$
a \leqq \arg m_{j} w_{j}<\mu+a+(p+1) \psi=a+\pi,
$$

from which it follows that $W=\sum_{j=1}^{n} m_{j} w_{j} \neq 0$. For, nonzero vectors drawn from the origin to points on the same side of a line through the origin cannot sum to zero. Since $W$ is the left side of equation (2.4) multiplied by $\left(Z_{1}-t_{1}\right)\left(Z_{2}-t_{2}\right) \cdots\left(Z_{p+1}-t_{p+1}\right)$, the assumption that at least $p+1$ zeros of $F(z)$ lie exterior to $S(K, \psi)$ has led to a contradiction of Theorem (2.1). In other words, at most $p$ zeros of $F(z)$ may lie exterior to $S(K, \psi)$.

4. Additional results on rational functions. To Theorem (1.1) may be added the following results.

THEOREM (4.1). If under the hypotheses of Theorem (1.1) not more than $n-2$ zeros of $F(z)$ are interior points of $S(K, \psi)$, then all the remaining zeros of $F(z)$ lie on the boundary of $S(K, \psi)$.

For, if the contrary were true, at least one zero $Z_{1}$ of $F(z)$ would lie exterior to $S(K, \psi)$ and $p$ additional zeros $Z_{2}, Z_{3}, \cdots, Z_{p+1}$ would lie either on the boundary of $S(K, \psi)$ or exterior to it. Inequality (3.1) would then hold for $k=1$, but less than $\psi$ would have to be replaced by not greater than $\psi$ for $k=2, \cdots, p+1$. However, inequality (3.2) remains valid and so again $W \neq 0$. That is, under the hypothesis of Theorem (4.1), not even one zero of $F(z)$ may lie exterior to $S(K, \psi)$.

To Theorem (1.1) may also be added the following theorem.

THEOREM (4.2). Let $R$ be any closed simply-connected region which consists only of points of the closed region $S(K, \psi)$ but is not identical with $S(K, \psi)$. 
Then not every function $F(z)$ of the form (1.1) has at most $p$ zeros exterior to $R$.

By definition the region $R$ omits at least one point $Z_{0}$ of the boundary of $S(K, \psi)$. At $Z_{0}, K$ subtends an angle of $\psi$; that is, there are in $K$ two points $t_{1}$ and $t_{2}$ such that $\psi=\arg \left(Z_{0}-t_{2}\right) /\left(Z_{0}-t_{1}\right)$. Let $Z_{0}-t_{1}=d_{1} e^{i \theta_{1}}$ and $Z_{0}-t_{2}$ $=d_{2} e^{i \theta_{2}}$ so that $\theta_{2}=\theta_{1}+\psi=\theta_{1}+[(\pi-\mu) /(p+1)]$. Then with $m_{1}=d_{1}^{p+1} e^{i \mu}$ and $m_{2}=d_{2}^{p+1}$ the function

$$
\begin{aligned}
F(z)=\int_{z_{0}}^{z} \int_{z_{0}}^{v_{p}} \cdots \int_{z_{0}}^{v_{3}} \int_{z_{0}}^{v_{2}}\left\{\left[m_{1} /\left(v_{1}-t_{1}\right)^{p+1}\right]\right. \\
\left.\quad+\left[m_{2} /\left(v_{1}-t_{2}\right)^{p+1}\right]\right\} d v_{1} d v_{2} \cdots d v_{p-1} d v_{p}
\end{aligned}
$$

is of the form (1.1) and the $m_{j}$ satisfy (1.2). Obviously,

$$
F\left(Z_{0}\right)=F^{\prime}\left(Z_{0}\right)=\cdots=F^{(p-1)}\left(Z_{0}\right)=0 .
$$

Furthermore,

$$
\begin{aligned}
F^{(p)}\left(Z_{0}\right) & =\left[m_{1} /\left(Z_{0}-t_{1}\right)^{p+1}\right]+\left[m_{2} /\left(Z_{0}-t_{2}\right)^{p+1}\right] \\
& =\left[d_{1}^{p+1} e^{i \mu} /\left(d_{1}^{p+1} e^{i(p+1) \theta_{1}}\right)\right]+\left[d_{2}^{p+1} /\left(d_{2}^{p+1} e^{i(p+1) \theta_{2}}\right)\right]=0 .
\end{aligned}
$$

That is, $F(z)$ has a zero of multiplicity $p+1$ at $Z_{0}$, a point exterior to $R$. Thus a function $F(z)$ of the form (1.1) has been exhibited with the property that it has more than $p$ zeros outside the region $R$, and thus the proof of Theorem (4.2) has been completed.

On the strength of Theorem (4.2), it may now be said that Theorem (1.1) furnishes the best possible result. That is, under the hypotheses of Theorem (1.1), $S(K, \psi)$ is the smallest region outside of which lie at most $p$ zeros of every function of type (1.1) satisfying (1.2).

As a consequence of Theorems (1.1) and (4.1) the following result may now be stated.

THEOREM (4.3). Let $K$ be the smallest closed convex region containing all $n$ of the distinct zeros of a polynomial $f(z)$ of degree $\nu$. Let $P(z)$ be an arbitrary polynomial of degree not exceeding $p-1$ and let $Q(z)=P(z) f(z)+f^{\prime}(z)$. Then at least $\nu-1$ zeros of $Q(z)$ lie in the closed star-shaped region $S_{1}=S(K, \pi /(p+1))$. If $Q(z)$ has, interior to $S_{1}$, at most $n-2$ zeros not counting those also zeros of $f(z)$, then no zero of $Q(z)$ lies exterior to $S_{1}$.

For, if $f(z)=\prod_{j=1}^{n}\left(z-z_{j}\right)^{m_{j}}$, then $Q(z) / f(z)$ has the form (1.1) with the $m_{j}$ as positive integers and thus $\mu=0$. Since the zeros of $Q(z)$ consist of the multiple zeros of $f(z)$ and the zeros of $Q(z) / f(z)$, the total number of zeros of $Q(z)$ in $S_{1}$ is not less than $(\nu-n)+(n-1)=\nu-1$. Similarly, the second part of the Theorem (4.3) follows at once from Theorem (4.1).

In the event that the rational function considered above has a zero instead of a pole at the point of infinity, the following result holds. 
THEOREM (4.4) Let $G(z)$ be a rational function which has at infinity a zero of order $p$ and has at the $n$ finite points $z_{j}(j=1,2, \cdots, n)$ simple poles with residues $m_{j}$ satisfying inequality (1.2). Let the poles $z_{j}$ be all contained in a convex region $K$ which subtends at the origin an angle $\delta, 0<\delta<(\pi-\mu) / p$. Then at least $n-1$ zeros of $G(z)$ lie in the region $S(K, \psi)$ where $\psi=(\pi-\mu-p \delta) /(p+2)$.

For, by hypothesis,

$$
\begin{aligned}
z^{p} G(z)= & \sum_{j=0}^{p} A_{j} z^{j}+\sum_{j=1}^{n}\left[m_{j} z^{p} /\left(z-z_{j}\right)\right] \\
=\sum_{j=0}^{p} A_{j} z^{j}+\sum_{j=1}^{n} m_{j} z_{j}^{p}\left\{\left[1 /\left(z-z_{j}\right)\right]\right. & \left.+\left(1 / z_{j}\right)+\left(z / z_{j}^{2}\right)+\cdots+\left(z^{p-1} / z_{j}^{p}\right)\right\} .
\end{aligned}
$$

If $Z_{1}, Z_{2}, \cdots, Z_{p+2}$ are any $p+2$ distinct zeros of $G(z)$, the coefficients $A_{j}$ may be eliminated from the equations $G\left(Z_{j}\right)=0, j=1,2, \cdots, p+2$, and the identity

$$
\sum_{j=1}^{n}\left[m_{j} z_{j}^{p} /\left(Z_{1}-z_{j}\right)\left(Z_{2}-z_{j}\right) \cdots\left(Z_{p+2}-z_{j}\right)\right]=0
$$

may thereby be obtained. By reasoning similar to that used in establishing Theorem (1.1), equation (4.1) leads to proof of Theorem (4.4).

5. Extension to meromorphic functions. Let

$$
\begin{aligned}
\phi(z, c) & =[1 /(z-c)]+(1 / c)+\left(z / c^{2}\right)+\cdots+\left(z^{q-1} / c^{q}\right), \quad q \leqq p, \\
\Phi_{n}(z) & =\left[m_{0} /\left(z-z_{0}\right)\right]+\sum_{j=0}^{p-1} A_{j} z^{j}+\sum_{j=1}^{n} m_{j} \phi\left(z, z_{j}\right) .
\end{aligned}
$$

Then, as is well known, $\Phi_{n}(z)$ as $n \rightarrow \infty$ converges to the meromorphic function

$$
\Phi(z)=m_{0} /\left(z-z_{0}\right)+\sum_{j=0}^{p-1} A_{j} z^{i}+\sum_{j=1}^{\infty} m_{j} \phi\left(z, z_{j}\right)
$$

absolutely and uniformly in every closed finite region not containing any points $z_{j}$, provided the series

$$
\sum_{j=1}^{\infty}\left|m_{j} / z_{i}^{q+1}\right|
$$

converges.

As the addition of the term $m_{0} /\left(z-z_{0}\right)$ to equation (1.1) and the replacement of the $A_{j}, j=1,2, \cdots, p-1$, by $\left(A_{j}+\sum_{k=1}^{n} m_{k} / z_{k}^{j+1}\right)$ will show, Theorem (2.2) remains valid if $F(z)$ is replaced by $\Phi_{n}(z)$. Consequently, if $Z_{1}^{(n)}, Z_{2}^{(n)}, \cdots, Z_{p}^{(n)}$ are any $p$ zeros of $\Phi_{n}(z)$, 


$$
\Phi_{n}(z)=\prod_{k=1}^{p}\left(Z_{k}^{(n)}-z\right) \sum_{j=0}^{n} m_{j} /\left[\left(z-z_{j}\right)\left(Z_{1}^{(n)}-z_{j}\right) \cdots\left(Z_{p}^{(n)}-z_{j}\right)\right] .
$$

Theorem (2.2) will now be extended to the meromorphic function $\Phi(z)$.

THEOREM (5.1). Let $R$ denote a finite set of finite closed regions which contain no pole of the meromorphic function $\Phi(z)$ defined by equation (5.1), but which contain in their interior $p$ zeros $Z_{1}, Z_{2}, \cdots, Z_{p}$ of $\Phi(z)$. Then, at any point $z$ of $R$,

$$
\Phi(z)=\prod_{k=1}^{p}\left(Z_{k}-z\right) \sum_{j=0}^{\infty}\left[m_{j} /\left(z-z_{j}\right)\left(Z_{1}-z_{j}\right) \cdots\left(Z_{p}-z_{j}\right)\right],
$$

the right side of this expression being absolutely and uniformly convergent in $R$.

In other words, if

$$
\Psi_{n}(z)=\prod_{j=1}^{p}\left(Z_{j}-z\right) \sum_{j=0}^{n} m_{j} /\left[\left(z-z_{j}\right)\left(Z_{1}-z_{j}\right) \cdots\left(Z_{p}-z_{j}\right)\right],
$$

it is to be proved that, given any positive $\epsilon$, one can find an $N>0$ so that $\left|\Phi(z)-\Psi_{n}(z)\right|<\epsilon$ for all $z$ in $R$, provided $n \geqq N$.

Without loss of generality it may be assumed

$$
\left|z_{0}\right| \leqq\left|z_{1}\right| \leqq\left|z_{2}\right| \leqq \cdots .
$$

Let $r_{u}, u=1,2, \cdots$, with $r_{\infty}=\infty$ be a sequence such that $0<r_{u}<\left|z_{u}\right|$. Let

$$
r_{u}=\lambda\left|z_{u}\right|, \quad 0<\lambda<1 .
$$

Let $u$ be taken so large that circle $C_{u}$ with center at $z=0$ and radius $r_{u}$ contains $R$ and

$$
\sum_{j=u}^{n}\left|m_{j} / z_{j}^{p+1}\right|<\left[(1-\lambda)^{p+1} / 3 r_{u}^{p} 2^{p+1}\right] \epsilon
$$

for all $n \geqq u$. Inequality (5.6) is possible because of the convergence of series (5.2).

Due to the uniform convergence of $\Phi_{n}(z)$ to $\Phi(z)$ in $R$, one can find an $N_{1} \geqq u$ so that

$$
\left|\Phi(z)-\Phi_{n}(z)\right|<\epsilon / 3
$$

for all $z$ in $R$, provided $n \geqq N_{1}$. Let circles $\Gamma_{k}$ be constructed with centers at $Z_{k}$ and with radii $\rho_{k}$ so small that the $\Gamma_{k}$ lie in $R$ but do not overlap one another. Then by Hurwitz' Theorem one can find an $N_{2} \geqq N_{1}$ so that $\Phi_{n}(z)$ has a zero $Z_{k}^{(n)}$ in each circle $\Gamma_{k}$ provided $n \geqq N_{2}$. Let the $\rho_{k}$ be taken so small that

$$
\tau_{j}=\prod_{k=1}^{p}\left[\left(Z_{k}-z\right) /\left(Z_{k}-z_{j}\right)\right]-\prod_{k=1}^{p}\left[\left(Z_{k}^{(n)}-z\right) /\left(Z_{k}^{(n)}-z_{j}\right)\right]
$$


for all $z$ in $R$ satisfies the inequality

$$
\left|\tau_{j}\right|<\epsilon / 3 M
$$$$
j=0,1, \cdots, u-1 \text {, }
$$

where $M=\max \left|\sum_{j=0}^{u-1} m_{j} /\left(z-z_{j}\right)\right|$, all $z$ in $R$.

Using (5.3) one may write

$$
\Psi_{n}(z)-\Phi_{n}(z)=\sigma_{1}+\sigma_{2}
$$

where

$$
\sigma_{1}=\sum_{j=0}^{u-1} m_{j} \tau_{j} /\left(z-z_{j}\right), \quad \sigma_{2}=\sum_{j=u}^{n} m_{j} \tau_{j} /\left(z-z_{j}\right) .
$$

From (5.8) it follows that

$$
\left|\sigma_{1}\right|<\epsilon / 3 \quad \text { for all } z \text { in } R \text {, if } n \geqq N_{2} \text {. }
$$

With respect to the terms in $\sigma_{2}$, it is to be noted that for all points $z$ and $z^{\prime}$ in $R$

$$
\left|z-z^{\prime}\right| \leqq 2 r_{w}
$$

and, by (5.5), that

$$
\left|z-z_{j}\right| \geqq\left|z_{j}\right|-r_{u}=\left|z_{j}\right|-\lambda\left|z_{u}\right| \geqq(1-\lambda)\left|z_{j}\right|
$$

for $j=u, u+1, \cdots$ Hence,

$$
\left|\sigma_{2}\right| \leqq\left[2^{p+1} r_{u}^{p} /(1-\lambda)^{p+1}\right] \sum_{j=u}^{n}\left|m_{j} / z_{j}^{p+1}\right|
$$

From (5.6) it may be inferred that

$$
\left|\sigma_{2}\right|<\epsilon / 3 \quad \text { for all } z \text { in } R \text {, if } n \geqq N_{2} \text {. }
$$

Finally from (5.7), (5.9), (5.10), and the relation

$$
\Phi(z)-\Psi_{n}(z)=\Phi(z)-\Phi_{n}(z)-\sigma_{1}-\sigma_{2},
$$

it follows that $\left|\Phi(z)-\Psi_{n}(z)\right|<\epsilon$ for all $z$ in $R$ if $n \geqq N=N_{2}$. Thus the proof of Theorem (5.1) has been completed.

From Theorem (5.1) the following generalization of Theorem (2.1) may be concluded.

TheOREM (5.2). Let $Z_{1}, Z_{2}, \cdots, Z_{p+1}$ denote any $p+1$ zeros of the function $\Phi(z)$ defined by equation (5.1). Then

$$
\sum_{j=0}^{\infty} m_{j} /\left[\left(Z_{1}-z_{j}\right)\left(Z_{2}-z_{j}\right) \cdots\left(Z_{p+1}-z_{j}\right)\right]=0 .
$$

Finally, from Theorem (5.2) the following generalization of Theorem (1.1) 
may be deduced.

THEOREM (5.3). Let all the $m_{j}$ in equation (5.1) satisfy inequality (1.2). Let $K$ be the smallest convex region which encloses all the poles $z_{j}$ of $\Phi(z)$ and let $S(K, \psi)$ denote the star-shaped region comprised of all points from which $K$ subtends an angle of at least $\psi=(\pi-\mu) /(p+1)$. Then $\Phi(z)$ has at most $p$ zeros exterior to $S(K, \psi)$.

The region $K$ of this theorem is in general infinite.

6. Applications to the derivative of entire functions of finite genre. Theorem (5.1) will now be applied to the derivative of entire functions $E(z)$ of genre $p$. Such functions may be written in the Weierstrass form

$$
E(z)=e^{P(z)} z^{r} \prod_{j=1}^{\infty}\left\{\left[1-\left(z / z_{j}\right)\right] \exp \sum_{k=1}^{p_{2}}(1 / k)\left(z / z_{j}\right)^{k}\right\}
$$

where $P(z)$ is a polynomial of degree $p_{1}$ and $p=\max \left(p_{1}, p_{2}\right)$. It will be convenient to assume $p_{1}=p_{2}=p$. The logarithmic derivative of $E(z)$ may be $\left.{ }^{7}\right)$ represented by the uniformly convergent series

$$
E^{\prime}(z) / E(z)=P^{\prime}(z)+(r / z)+\sum_{j=1}^{\infty} \phi\left(z, z_{j}\right)
$$

and thus has the form (5.1) with $z_{0}=0, m_{0}=r$, all other $m_{j}=1$, and thus $\mu=0$ and $\psi=\pi /(p+1)$.

Thus the following result may be concluded from Theorem (5.1).

THEOREM (6.1). Let $E(z)$, an entire function of genre $p$, have the zeros $z_{0}=0, z_{1}, z_{2}, \cdots$ with the multiplicities $m_{0}, m_{1}, m_{2}, \cdots$ respectively; let $Z_{1}, Z_{2}, \cdots, Z_{p}$ denote any $p$ zeros of its derivative $E^{\prime}(z)$. Then

$$
E^{\prime}(z)=E(z) \sum_{j=0}^{\infty}\left\{\left[m_{j} /\left(z-z_{j}\right)\right] \prod_{k=1}^{p}\left[\left(Z_{k}-z\right) /\left(Z_{k}-z_{j}\right)\right]\right\} .
$$

Likewise, Theorem (5.2) applies without change to the function $E^{\prime}(z)$ and Theorem (5.3) applies to $E^{\prime}(z)$ on taking $\psi=\pi /(p+1)$.

In particular, if the convex region $K$ is taken as the sector $|\arg z| \leqq \alpha$ $<\pi /(p+1)$, the region complementary to $S(K, \pi /(p+1))$ is the sector $|\arg (-z)|<[\pi /(p+1)]-\alpha$. Since the latter sector has no points in common with the former sector, it contains only zeros of $E^{\prime}(z)$ which are not zeros of $E(z)$. From Theorem (5.3) the following result may be then concluded.

THEOREM (6.2). Let $\alpha$ and $\beta$ be any two non-negative real numbers such that $\alpha \leqq \beta$ and $\alpha+\beta=\pi /(p+1)$ and let $E(z)$ be an entire function of genre $p$. Then, if all the zeros of $E(z)$ lie in the sector $|\arg z| \leqq \alpha$, at most $p$ zeros of $E^{\prime}(z)$ will lie in the sector $|\arg (-z)|<\beta$.

( $\left.{ }^{7}\right)$ E. Borel, Leçons sur les fonctions entières, Paris, 1921, pp. 36-44. 
For example, if $p=0$, Theorem (6.1) yields the result that, if all the zeros of an entire function of genre zero lie in a sector with an aperture not exceeding $\pi$, then all the zeros of its derivative also lie in the same sector. This extends to entire functions of genre zero the Lucas' Theorem that any convex region enclosing all the zeros of a polynomial also encloses all the zeros of the derivative. This extension seems to have been first made by $\operatorname{Porter}\left({ }^{8}\right)$.

Another interesting special choice of region $K$ is that of a semi-infinite strip. In that case, the result may be stated as follows.

THEOREM (6.3). If all the zeros of the entire function $E(z)$ of genre $p$ lie in the closed strip $A^{\prime} A B B^{\prime}$ (see the accompanying figure), then at most $p$ zeros of $E^{\prime}(z)$ lie in the open sector $C^{\prime} C D D^{\prime}$ (the exterior of $A^{\prime} A B B^{\prime}$ if $p=0$ ).

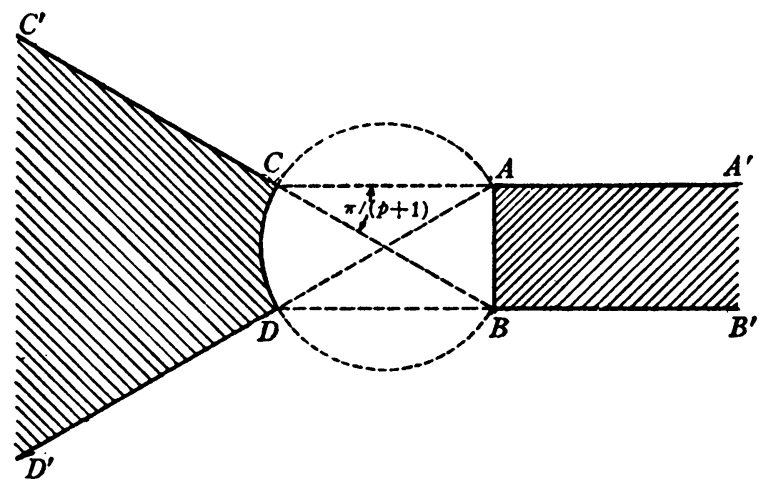

Finally, as an application of Theorem (5.2) the following result will be derived.

THEOREM (6.4). Let $E(z)$, a real entire function of genre $p$, have only real zeros. Let $N$ be the number of (real and non-real) zeros which $E^{\prime}(z) / E(z)$ has in excess of one between each pair of successive zeros of $E(z)$. Then $N \leqq p$.

Let the point-set $R$ be formed by choosing exactly one real zero of $E^{\prime}(z) / E(z)$ between each pair of successive zeros of $E(z)$. The existence of $R$ is assured by Rolle's Theorem. Let $E^{\prime}(z) / E(z)$ have exterior to $R$ the zeros $Z_{1}, Z_{2}, \cdots, Z_{p+1}$. These fall into three types:

(1) the real zeros $Z_{1}, Z_{2}, \cdots, Z_{a}, a \leqq p+1$, which lie on the same interval $I$ of the real axis as do the zeros of $E(z)$;

(2) the real zeros $Z_{a+1}, Z_{a+2}, \cdots, Z_{b}, b \leqq p+1$, which lie exterior to $I$, and

(3) the non-real zeros $Z_{b+1}, Z_{b+2}, \cdots, Z_{p+1}$.

The corresponding factors in equation (5.11) will now be considered.

(8) M. B. Porter, On a theorem of Lucas, Proc. Nat. Acad. Sci. U.S.A. vol. 2 (1916) pp. 247$248,335-336$. 
First, by Rolle's Theorem, $E^{\prime}(z) / E(z)$ has an odd number of zeros between each pair of successive zeros $z_{j}$ of $E(z)$, and hence, in excess of the one zero already counted, it has an even number of zeros or no zero between each pair of successive zeros of $E(z)$. For example, if $E^{\prime}(z) / E(z)$ had the excess zeros $Z_{1}, Z_{2}, \cdots, Z_{2 k}, 2 k \leqq a$, between the two successive zeros $z_{1}$ and $z_{2}$ of $E(z)$, then $\left(Z_{1}-z_{j}\right)\left(Z_{2}-z_{j}\right) \cdots\left(Z_{2 k}-z_{j}\right)>0$ for all $j$. Furthermore, the factor $Z_{i}-z_{j}$ corresponding to any zero of the second type $(i=a+1, a+2, \cdots, b)$ will have the same sign for all $j$. Finally, since $E^{\prime}(z)$ is real, the zeros $Z_{i}$ of the third type $(i=b+1, \cdots, p+1)$ fall into conjugate imaginary pairs. Corresponding to such a pair, the product $\left(Z_{i}-z_{j}\right)\left(\bar{Z}_{i}-z_{j}\right)>0$ for all $j$. In short, since all terms in (5.11) have the same sign, their sum cannot vanish. Hence, the assumption that $E^{\prime}(z) / E(z)$ has at least $p+1$ zeros $Z_{j}$ exterior to $R$ has led to a contradition. Consequently, $N \leqq p$, as was to be proved.

Theorem (6.4) is the special case $\nu=0$ of the Laguerre-Borel Theorem $\left({ }^{7}\right)$ : If the real entire function $E(z)$ of genre $p$ has exactly $\nu$ non-real zeros, then its derivative has at most $p+\nu$ real and non-real zeros in excess of one between each pair of successive zeros of $E(z)$.

It appears that this theorem can be proved in a manner similar to Theorem (6.4). For, if $Z_{1}, Z_{2}, \cdots, Z_{p+\nu+1}$ are $p+\nu+1$ distinct zeros of $E^{\prime}(z) / E(z)$, then $\nu$ of the $z_{j}$ may be eliminated from the $\nu+1$ equations obtained from equation $(5.11)$ by replacing $Z_{p+1}$ by $Z_{p+i}, i=1,2, \cdots, \nu+1$. It may be conjectured that the resulting identity connecting the $Z_{1}, Z_{2}, \cdots$, $Z_{p+p+1}$ with the remaining $z_{j}$ will be in the form of equation (5.11). If the remaining $z_{j}$ are taken as the real $z_{j}$, the reasoning used for Theorem (6.4) should then lead to a proof of the Laguerre-Borel Theorem. The same identity should also lead to a generalization of Theorem (6.4) giving, in the case of a real or complex entire function $E(z)$ of genre $p$, the location of all but $p+\nu$ zeros of $E^{\prime}(z)$ when the location of all but $\nu$ zeros of $E(z)$ is known. However, details along these lines have not as yet been carried to completion.

In conclusion, it may be pointed out that Theorems (6.1), (6.2), (6.3), and (6.4) remain valid if $E^{\prime}(z)$ is replaced by the function $H(z)=E^{\prime}(z)+Q(z) E(z)$ where $Q(z)$ is an arbitrary polynomial of degree at most $p-1$. (In the case of Theorem (6.4), $Q(z)$ must also be assumed to be real.) This may be proved in either of two ways. First, $H(z) / E(z)$ is a meromorphic function of the form $\Phi(z)$ with all the $m_{j}$ real so that, in satisfying Theorems (5.1), (5.2), and (5.3), it also satisfies Theorems (6.1) to (6.4). Alternatively, $H(z)$ has the same zeros as the derivative of the function $E_{1}(z)=E(z) \exp \int Q(z) d z$, which is an entire function of genre $p$ with the same zeros as $E(z)$; hence, the theorems concerning the zeros of $H(z)$ are the same as those concerning the zeros of $E_{1}^{\prime}(z)$.

UNIVERSITY OF WISCONSIN, MiLWAUKeE, Wis. 